\title{
Numerical investigation on three treatments for eliminating the singularities of acoustic fundamental solutions in the singular boundary method
}

\author{
Zhuo-Jia Fu, Wen Chen \& Wenzhen Qu \\ College of Mechanics and Materials and State Key Laboratory of \\ Hydrology-Water Resources and Hydraulic Engineering, \\ Hohai University, P. R. China
}

\begin{abstract}
This study considers the numerical comparison on three treatments for eliminating the singularities of acoustic fundamental solutions in singular boundary method (SBM). The singular boundary method is a recent meshless boundary collocation method, which introduces the concept of source intensity factors to eliminate the singularity of the fundamental solutions. Recently, three treatments, the inverse interpolation technique (IIT), the semi-analytical technique with boundary IIT (SAT1) and the semi-analytical technique with integral mean value (SAT2), have been proposed to determine the source intensity factors for removing the singularities of acoustic fundamental solutions at origin. This study compares numerical efficiency and stability of these three approaches on two benchmark examples under two-dimensional exterior acoustic problems.

Keywords: boundary collocation, singular boundary method, source intensity factors, singularity, acoustic fundamental solution.
\end{abstract}

\section{Introduction}

The singular boundary method (SBM) [1] is a recent meshless boundary collocation method, which introduces the concept of source intensity factor to regularize the singularities of fundamental solutions, in some literature it is also called the origin intensity factor. The SBM avoids singular numerical integrals in 
the boundary element method and circumvents the troublesome placement of the fictitious boundary in the method of fundamental solutions.

In the SBM, an inverse interpolation technique (IIT) has been first proposed to determine the above-mentioned source intensity factors of fundamental solutions. This SBM formulation has been successfully applied to interior and exterior Laplace [2-4], Poisson [5], Helmholtz [6] and elastostatic [7] problems. Later, $\mathrm{Gu}$ et al. [8] introduced the desingularization of the subtracting and adding-back technique and proposed an improved singular boundary method (ISBM) for interior and exterior potential problems. Its main improvement is developing a semi-analytical technique (SAT1) to determine the source intensity factors without any inner sample nodes. The approach employs the null-field integral equations to evaluate the source intensity factors of Laplace fundamental solution on Neumann boundary. And then it uses the inverse interpolation technique with boundary source points to determine the source intensity factors of Laplace fundamental solution on a Dirichlet boundary. Then Fu et al. [9-11] used the relationships between Laplace and Helmholtz fundamental solutions to extend the ISBM to solve Helmholtz equations. Recently, another semianalytical technique (SAT2) has been proposed [12]. The SAT2 introduces the integral mean value approach to determine the source intensity factors of Laplace fundamental solutions on Dirichlet boundary.

This study will extend the SAT2 to determine the source intensity factors of the Helmholtz fundamental solutions, and then compares numerical accuracy and stability of these three approaches (IIT, SAT1 and SAT2) on 2D exterior acoustic problems. A brief outline of the paper is as follows. Section 2 describes three treatments for eliminating the singularities of acoustic fundamental solutions in the singular boundary method. In Section 3, the efficiency and accuracy of these three approaches are examined in 2D benchmark examples. Finally, Section 4 concludes this paper with some remarks.

\section{Three ways to calculate the source intensity factors in SBM}

The problem under consideration is the propagation of time-harmonic acoustic waves in a homogeneous isotropic acoustic medium $D$ exterior to a closed bounded curve $\Gamma$, which can be described by the Helmholtz equation

$$
\nabla^{2} u(x)+k^{2} u(x)=0, \quad x \in D,
$$

subjected to the boundary conditions

$$
\begin{gathered}
u(x)=\bar{u} \quad x \in \Gamma_{D}, \\
q(x)=\frac{\partial u(x)}{\partial \mathbf{n}}=\bar{q} \quad x \in \Gamma_{N},
\end{gathered}
$$


where $k=\omega / c$ the wavenumber, $\omega$ the angular frequency, $c$ the wave speed in the exterior acoustic medium $D$, and $\mathbf{n}$ the unit outward normal on physical boundary. $\Gamma_{D}$ and $\Gamma_{N}$ represent the essential boundary (Dirichlet) and the natural boundary (Neumann) conditions, respectively, which construct the whole closed bounded curve $\Gamma=\Gamma_{D}+\Gamma_{N}$, and $u$ is complex-valued amplitude of radiated and/or scattered wave (velocity potential or acoustic pressure)

$$
u= \begin{cases}u_{R}=u_{T}, & \text { if radiation, } \\ u_{S}=u_{T}-u_{I}, & \text { if scattering } \\ u_{R+S}=u_{T}-u_{I}, & \text { if both }\end{cases}
$$

where the subscripts $T, R$ and $I$ denote the total, radiation and incidence wave, respectively. For the exterior acoustic problems, it requires guaranteeing the physical requirement that all scattered and radiated waves are outgoing. This is accomplished by imposing an appropriate radiation condition at infinity, which is termed the Sommerfeld radiation condition:

$$
\lim _{r \rightarrow \infty} r^{\frac{1}{2}(\operatorname{dim}-1)}\left(\frac{\partial u}{\partial r}-i k u\right)=0
$$

where $\operatorname{dim}$ is the dimension of the acoustic problems, and $i=\sqrt{-1}$. By utilizing single layer fundamental solutions, the SBM approximate solutions $u(x)$ and $q(x)$ of exterior acoustic problem (Eqs. (1) and (2)) can be expressed as follows

$$
\begin{gathered}
u\left(x_{m}\right)=\left\{\begin{array}{l}
\sum_{j=1}^{N} \alpha_{j} G\left(x_{m}, s_{j}\right), \quad x_{m} \in D \backslash \Gamma \\
\sum_{j=1, j \neq m}^{N} \alpha_{j} G\left(x_{m}, s_{j}\right)+\alpha_{m} U_{S}^{j j}, x_{m} \in \Gamma
\end{array}\right. \\
q\left(x_{m}\right)=\frac{\partial u\left(x_{m}\right)}{\partial \mathbf{n}_{x}}=\left\{\begin{array}{l}
\sum_{j=1}^{N} \alpha_{j} \frac{\partial G\left(x_{m}, s_{j}\right)}{\partial \mathbf{n}_{x}}, \quad x_{m} \in D \backslash \Gamma \\
\sum_{j=1, j \neq m}^{N} \alpha_{j} \frac{\partial G\left(x_{m}, s_{j}\right)}{\partial \mathbf{n}_{x}}+\alpha_{m} Q_{S}^{j j}, x_{m} \in \Gamma
\end{array},\right.
\end{gathered}
$$

where $N$ denotes the number of source points $s_{j}, \alpha_{j}$ the $j$ th unknown coefficient, $\mathbf{n}_{x}$ the outward normal unit vector on the collocation points $x_{m}$, the $2 \mathrm{D}$ 
fundamental solutions $G\left(x_{m}, s_{j}\right)=i H_{0}^{(1)}\left(k r_{m j}\right) / 4, H_{n}^{(1)}$ is the $n$th order Hankel function of the first kind, the Euclidean distance $r_{m j}=\left\|x_{m}-s_{j}\right\|_{2}$. If the collocation points and source points coincide, i.e., $x_{m}=s_{j}$, the well-known singularities are encountered. The SBM introduces the concept of the source intensity factors $U_{S}^{i j}$ and $Q_{S}^{j j}$ to avoid these singularities. Its key issue is how to determine these source intensity factors $U_{S}^{j j}$ and $Q_{S}^{j j}$. Fortunately, it is of interest to point out that the fundamental solutions of Helmholtz equation have the similar order of the singularities as the related fundamental solutions of Laplace equation [13]. The corresponding relationships can be represented by the following asymptotic expressions

$$
\begin{gathered}
G\left(x_{m}, s_{j}\right)=G_{0}\left(x_{m}, s_{j}\right)-\frac{1}{2 \pi}\left(\ln \left(\frac{k}{2}\right)+\gamma-\frac{i \pi}{2}\right), \quad r_{m j} \rightarrow 0, \\
\frac{\partial G\left(x_{m}, s_{j}\right)}{\partial \mathbf{n}_{x}}=\frac{\partial G_{0}\left(x_{m}, s_{j}\right)}{\partial \mathbf{n}_{x}}, \quad r_{m j} \rightarrow 0, \\
\frac{\partial G\left(x_{m}, s_{j}\right)}{\partial \mathbf{n}_{s}}=\frac{\partial G_{0}\left(x_{m}, s_{j}\right)}{\partial \mathbf{n}_{s}}, \quad r_{m j} \rightarrow 0,
\end{gathered}
$$

where the Euler constant $\gamma=0.57721566490153286, \mathbf{n}_{s}$ the outward normal unit vector on the source points $s_{j}$, the $2 \mathrm{D}$ fundamental solution of the Laplace equation $G_{0}=-\ln \left(r_{m j}\right) /(2 \pi)$. Hence, we can introduce the existing approaches to determine the source intensity factors for the Laplace equation, and then implement the above-mentioned relationship to calculate the source intensity factors for the Helmholtz equation. In the following section, we will introduce three approaches to determine the source intensity factors for removing the singularities of acoustic fundamental solutions at origin.

\subsection{Inverse interpolation technique}

This section will introduce a simple numerical technique, called the inverse interpolation technique (IIT) [3, 6], to determine the source intensity factors for Laplace equations. Then we can use the relationships (4) to determine the source intensity factors for Helmholtz equations. In the first step, the IIT requires choosing a known sample solution $u_{S 0}$ of Laplace equations and placing some sample points $y_{k}$ inside the physical domain. It is noted that the sample points $y_{k}$ do not coincide with the source points $s_{j}$, and the sample points number $N K$ should not be fewer than the source node number $N$ on the physical boundary. 
By using the interpolation formula (3), we can then determine the influence coefficients $\beta_{j}$ and $\bar{\beta}_{j}$ by solving the following linear equations

$$
\begin{gathered}
\left\{G_{0}\left(y_{k}, s_{j}\right)\right\}\left\{\beta_{j}\right\}=\left\{u_{S 0}\left(y_{k}\right)\right\}, \\
\left\{\frac{\partial G_{0}\left(y_{k}, s_{j}\right)}{\partial \mathbf{n}_{x}}\right\}\left\{\bar{\beta}_{j}\right\}=\left\{\frac{\partial u_{S 0}\left(y_{k}\right)}{\partial \mathbf{n}_{x}}\right\} .
\end{gathered}
$$

Replacing the sample points $y_{k}$ with the boundary collocation points $x_{m}$, the SBM interpolation matrix (Eqs. (1) and (2)) can be written as

$$
\begin{gathered}
{\left[\begin{array}{cccc}
U_{S 0}^{11} & G_{0}\left(x_{1}, s_{2}\right) & \cdots & G_{0}\left(x_{1}, s_{N}\right) \\
G_{0}\left(x_{2}, s_{1}\right) & U_{S 0}^{22} & \cdots & G_{0}\left(x_{2}, s_{N}\right) \\
\vdots & \vdots & \ddots & \vdots \\
G_{0}\left(x_{N}, s_{1}\right) & G_{0}\left(x_{N}, s_{2}\right) & \cdots & U_{S 0}^{N N}
\end{array}\right]\left\{\beta_{j}\right\}=\left\{u_{S 0}\left(x_{m}\right)\right\}} \\
{\left[\begin{array}{cccc}
\frac{\partial G_{0}\left(x_{1}, s_{2}\right)}{\partial \mathbf{n}_{x}} & \cdots & \frac{\partial G_{0}\left(x_{1}, s_{N}\right)}{\partial \mathbf{n}_{x}} \\
\frac{\partial G_{0}\left(x_{2}, s_{1}\right)}{\partial \mathbf{n}_{x}} & Q_{S 0}^{22} & \cdots & \frac{\partial G_{0}\left(x_{2}, s_{N}\right)}{\partial \mathbf{n}_{x}} \\
\vdots & \vdots & \ddots & \vdots \\
\frac{\partial G_{0}\left(x_{N}, s_{1}\right)}{\partial \mathbf{n}_{x}} & \frac{\partial G_{0}\left(x_{N}, s_{2}\right)}{\partial \mathbf{n}_{x}} & \cdots & Q_{S 0}^{N N}
\end{array}\right]\left\{\bar{\beta}_{j}\right\}=\left\{\frac{\partial u_{S 0}\left(x_{m}\right)}{\partial \mathbf{n}_{x}}\right\}}
\end{gathered}
$$

Then the source intensity factors for the Laplace equation can be calculated by

$$
\begin{gathered}
U_{S 0}^{m m}=\left(u_{S 0}\left(x_{m}\right)-\sum_{j=1, s_{j} \neq x_{m}}^{N} \beta_{j} G_{0}\left(x_{m}, s_{j}\right)\right) / \beta_{m} \quad x_{m}=s_{j}, x_{m} \in \Gamma_{D} \\
Q_{S 0}^{m m}=\left(\frac{\partial u_{S 0}\left(x_{m}\right)}{\partial \mathbf{n}_{x}}-\sum_{j=1, s_{j} \neq x_{m}}^{N} \beta_{j} \frac{\partial G_{0}\left(x_{m}, s_{j}\right)}{\partial \mathbf{n}_{x}}\right) / \bar{\beta}_{m} \quad x_{m}=s_{j}, x_{m} \in \Gamma_{N^{*}}(7 b)
\end{gathered}
$$

Hence the source intensity factors for the Helmholtz equation can be represented as 


$$
\begin{gathered}
U_{S}^{m m}=U_{S 0}^{m m}-\frac{1}{2 \pi}\left(\ln \left(\frac{k}{2}\right)+\gamma-\frac{i \pi}{2}\right), x_{m}=s_{j}, x_{m} \in \Gamma_{D} \\
Q_{S}^{m m}=Q_{S 0}^{m m}, \quad x_{m}=s_{j}, x_{m} \in \Gamma_{N} .
\end{gathered}
$$

\subsection{Semi-analytical technique with boundary IIT (SAT1)}

This section will introduce a semi-analytical technique $[8,9]$ to calculate the source intensity factors.

\subsubsection{Source intensity factors on Neumann boundary conditions}

By adopting the subtracting and adding-back technique in Eq. (3b) at $x_{m}=s_{j}$, we obtain

$$
\begin{aligned}
& q\left(x_{m}\right)=\frac{\partial u\left(x_{m}\right)}{\partial \mathbf{n}_{x}}=\sum_{j=1}^{N} \alpha_{j} \frac{\partial G\left(x_{m}, s_{j}\right)}{\partial \mathbf{n}_{x}} \\
& \quad=\sum_{j=1}^{N}\left(\alpha_{j}-\alpha_{m} \Pi_{j m}\right) \frac{\partial G\left(x_{m}, s_{j}\right)}{\partial \mathbf{n}_{x}}+\alpha_{m} \sum_{j=1}^{N} \Pi_{j m}\left(\frac{\partial G\left(x_{m}, s_{j}\right)}{\partial \mathbf{n}_{x}}-\frac{\partial G_{0}\left(x_{m}, s_{j}\right)}{\partial \mathbf{n}_{x}}\right) \\
& \quad+\alpha_{m} \sum_{j=1}^{N} \Pi_{j m}\left(\frac{\partial G_{0}\left(x_{m}, s_{j}\right)}{\partial \mathbf{n}_{x}}+\frac{\partial G_{0}^{I}\left(x_{m}, s_{j}\right)}{\partial \mathbf{n}_{s}}\right)-\alpha_{m} \sum_{j=1}^{N} \Pi_{j m} \frac{\partial G_{0}^{I}\left(x_{m}, s_{j}\right)}{\partial \mathbf{n}_{s}},
\end{aligned}
$$

where $G_{0}^{I}\left(x_{m}, s_{j}\right)$ denotes the fundamental solution of the interior Laplace equation, $\Pi_{j m}=L_{j} / L_{m}$, in which $L_{j}$ is half length of the curve $\widehat{s_{j-1} s_{j+1}}$ between source points $s_{j-1}$ and $s_{j+1}$ as shown in Fig. 1. Note that $\Pi_{m m}=1$.

According to the dependency of the outward normal vectors on the fundamental solutions of interior and exterior Laplace equations [8, 14], we have the following relationships

$$
\begin{cases}\frac{\partial G_{0}\left(x_{m}, s_{j}\right)}{\partial \mathbf{n}_{s}}=-\frac{\partial G_{0}^{I}\left(x_{m}, s_{j}\right)}{\partial \mathbf{n}_{s}}, & x_{m} \neq s_{j} \\ \frac{\partial G_{0}\left(x_{m}, s_{j}\right)}{\partial \mathbf{n}_{s}}=\frac{\partial G_{0}^{I}\left(x_{m}, s_{j}\right)}{\partial \mathbf{n}_{s}}, & x_{m}=s_{j}\end{cases}
$$

and 


$$
\begin{gathered}
\lim _{s_{j} \rightarrow x_{m}}\left(\frac{\partial G_{0}\left(x_{m}, s_{j}\right)}{\partial \mathbf{n}_{x}}+\frac{\partial G_{0}\left(x_{m}, s_{j}\right)}{\partial \mathbf{n}_{s}}\right)=0, \\
V_{m}=-\frac{1}{L_{m}}=\sum_{j=1}^{N} \Pi_{j m} \frac{\partial G_{0}^{I}\left(x_{m}, s_{j}\right)}{\partial \mathbf{n}_{s}} .
\end{gathered}
$$

If the boundary shape is of a straight line, Eq. (10b) is explicitly equal to zero since $\mathbf{n}_{x}\left(x_{m}\right)=\mathbf{n}_{s}\left(s_{j}\right)$ at all boundary knots. For an arbitrarily shaped smooth boundary, herein we assume that the source point $s_{j}$ approaches inchmeal to the collocation point $x_{m}$ along a line segment, then Eq. (10b) is tenable. In addition, Eq. (10c) is derived based on the discretization of the reduced full-fields equations [15]. With the help of Eqs. (4) and (10), Eq. (9) can be regularized as

$$
q\left(x_{m}\right)=\sum_{j=1, j \neq m}^{N} \alpha_{j} \frac{\partial G\left(x_{m}, s_{j}\right)}{\partial \mathbf{n}_{x}}-\alpha_{m}\left(\sum_{j=1, j \neq m}^{N} \Pi_{j m} \frac{\partial G_{0}\left(x_{m}, s_{j}\right)}{\partial \mathbf{n}_{s}}+V_{m}\right)
$$

By contrast with Eq. (3b) at $x_{m}=s_{j}$, we can obtain

$$
Q_{S}^{j j}=Q_{S 0}^{j j}=-V_{m}-\sum_{j=1, j \neq m}^{N} \Pi_{j m} \frac{\partial G_{0}\left(x_{m}, s_{j}\right)}{\partial \mathbf{n}_{s}}
$$

which is the source intensity factors for Neumann boundary conditions in Eq. (3b). Unlike the first treatment, this method does not require the additional inner sample nodes.

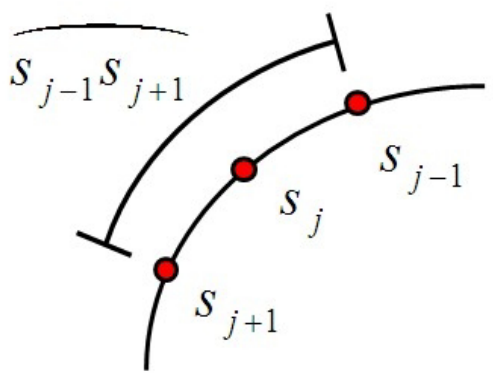

Figure 1: Schematic configuration of source points $s_{j}$ and the related curve $\widehat{s_{j-1} s_{j+1}}$. 


\subsubsection{Source intensity factors on Dirichlet boundary conditions}

Then the source intensity factors $U_{S 0}^{j j}$ can be calculated by the inverse interpolation technique $[8,11]$. This strategy chooses a sample solution $\bar{u}_{0}$ of $2 \mathrm{D}$ Laplace equation, e.g., $\bar{u}_{0}=x+y+c$, then $2 N+1$ linear equations are obtained with $2 N+1$ unknowns $\left(U_{0}^{j j}, \beta_{j}, c\right)$ on $N$ boundary source points and one inner point $x_{I}$.

$$
\begin{gathered}
\bar{u}_{0}\left(x_{m}\right)=\sum_{j=1, j \neq m}^{N} \beta_{j} G_{0}\left(x_{m}, s_{j}\right)+\beta_{m} U_{0}^{j j}+c, x_{m}=s_{j}, \\
\frac{\partial \bar{u}_{0}\left(x_{m}\right)}{\partial \mathbf{n}_{x}}=\sum_{j=1, j \neq m}^{N} \beta_{j} \frac{\partial G_{0}\left(x_{m}, s_{j}\right)}{\partial \mathbf{n}_{x}}+\beta_{m} Q_{0}^{j j}, x_{m}=s_{j}, \\
\bar{u}_{0}\left(x_{I}\right)=\sum_{j=1}^{N} \beta_{j} G_{0}\left(x_{I}, s_{j}\right)+c, x_{I} \neq s_{j} .
\end{gathered}
$$

Therefore, the source intensity factors $U_{S}^{j j}$ in Eq. (3a) can be determined indirectly by calculating the source intensity factors $U_{S 0}^{j j}$ by using Eq. (8a).

\subsection{Semi-analytical technique with integral mean value (SAT2)}

This section will introduce a recently developed semi-analytical technique [12], which does not require the inverse interpolation technique. As with Section 2.2.1, the regularized SBM formulation for Neumann boundary conditions (3b) can be expressed as follows

$$
q\left(x_{m}\right)=\sum_{j=1, j \neq m}^{N} \alpha_{j} \frac{\partial G\left(x_{m}, s_{j}\right)}{\partial \mathbf{n}_{x}}-\alpha_{m}\left(\sum_{j=1, j \neq m}^{N} \Pi_{j m} \frac{\partial G_{0}\left(x_{m}, s_{j}\right)}{\partial \mathbf{n}_{s}}+V_{m}\right)
$$

And

$$
Q_{S}^{j j}=Q_{S 0}^{j j}=-V_{m}-\sum_{j=1, j \neq m}^{N} \Pi_{j m} \frac{\partial G_{0}\left(x_{m}, s_{j}\right)}{\partial \mathbf{n}_{s}},
$$

is the aforementioned source intensity factors for Neumann boundary conditions. Next the regularized expressions for Dirichlet boundary conditions (3a) can be performed using Sarler's strategy [16], where the corresponding source intensity 
factors are directly set as an average value of Laplace fundamental solution over line segments. This can be formulated as

$$
U_{S 0}^{j j}=\frac{1}{L_{m}} \int_{\Gamma_{s}}\left(G_{0} x_{m}, s_{j}\right) d \Gamma_{s}=-\frac{1}{2 \pi L_{m}} \int_{\Gamma_{s}} \ln \left\|x_{m}-s_{j}\right\|_{2} d \Gamma_{s}, x_{m}=s_{j} .
$$

Then the source intensity factors $U_{S}^{j j}$ for Dirichlet boundary conditions can be calculated by using Eq. (8a).

\section{Numerical results}

In this section, the efficiency, accuracy and convergence of the above-mentioned three treatments (IIT, SAT1 and SAT2) in the SBM are implemented to solve 2D exterior acoustics problems. The numerical accuracy is measured by the relative root mean square errors (RMSE) $\operatorname{Lerr}(u)$, which is defined as

$$
\operatorname{Lerr}(u)=\sqrt{\frac{1}{N T} \sum_{k=1}^{N T}|u(k)-\bar{u}(k)|^{2}} / \sqrt{\frac{1}{N T} \sum_{k=1}^{N T}|\bar{u}(k)|^{2}},
$$

where $\bar{u}(k)$ and $u(k)$ are the analytical and numerical solutions at $x_{i}$, respectively, and $N T$ is the total number of test points in the interest domain. Unless otherwise specified, the inner sample nodes $y_{k}$ are uniform angular distribution on the circle with radius $\left(1-2 / L_{k}\right)$ and the inner additional point $x_{I}=(0.5,0.5)$ for the SAT1 in all the following numerical cases.

Example 1: Scattering problem of a soft infinite circular cylinder (Dirichlet boundary condition).

We consider a plane wave $e^{i k r \cos \theta}$ scattered by a soft infinite circular cylinder as shown in Fig. 2(a). The analytical solution of the scattering field $u_{S}$ [17] is

$$
u_{S}(r, \theta)=-\frac{J_{0}(k a)}{H_{0}^{(1)}(k a)} H_{0}^{(1)}(k r)-2 \sum_{n=1}^{\infty} i^{n} \frac{J_{n}(k a)}{H_{n}^{(1)}(k a)} H_{n}^{(1)}(k r) \cos n \theta .
$$

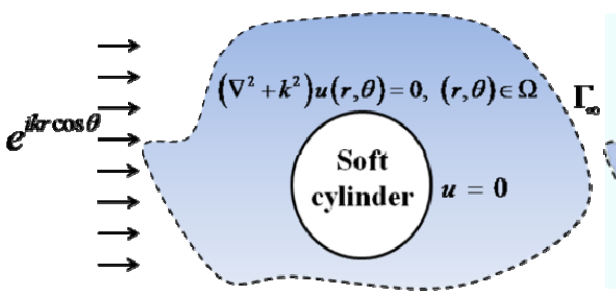

(a)

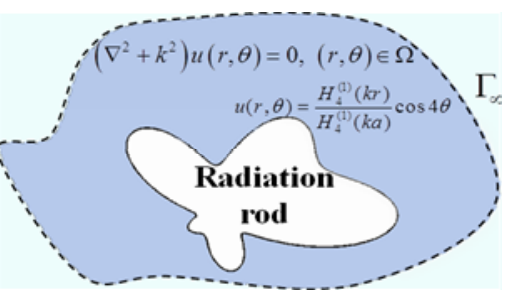

(b)

Figure 2: $\quad$ Sketch of (a) the scattering problem for a soft infinite cylinder and (b) the radiation problem of a soft infinite irregular-shaped rod. 


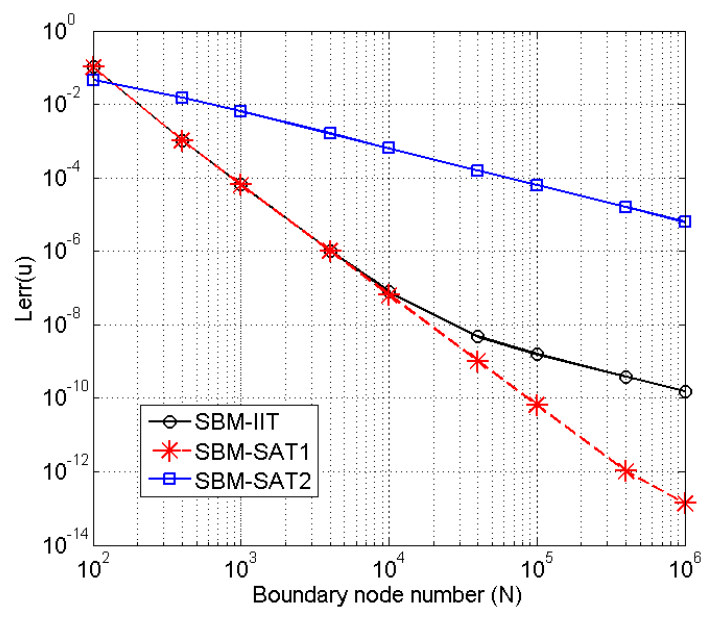

Figure 3: Convergence analysis $\operatorname{Lerr}(u)$ of the SBM with IIT, SAT1 and SAT2 for the scattering problem of a soft infinite cylinder with $k a=40$.

Fig. 3 shows the error analysis of the SBM with three treatments for the 2D scattering problem with $k a=40$. The analytical solutions in this case are calculated by using the first 100 terms in the above series representation (18). The test points $(N T=101)$ are uniform angular distribution on the circle with radius 1.2 . It can be found that all of these three methods converge with the increasing boundary node number $N$. In this case, under the same number of boundary knots, the SBM with SAT1 provides better results than the SBM with IIT and SAT2, and the slope of its convergence curve is about -3 . Moreover, the SBM with SAT2 has the slowest convergence rate, whose slope is about -1 . While the SBM with IIT has the same convergence rate to the SAT1 with a modestly increasing boundary node number $(N=10000)$, but it converges slowly with a further increasing boundary node number. This may result from the numerical instabililty of the IIT to calculate the source intensity factors.

Example 2: Radiation model for a soft infinite irregular-shaped rod (Dirichlet boundary condition).

Consider the radiation problem of a soft infinite irregular-shaped rod as shown in Fig. 2b. The analytical solution of the radiation field $u_{R}$ is

$$
u_{R}(r, \theta)=\frac{H_{4}^{(1)}(k r)}{H_{4}^{(1)}(k a)} \cos 4 \theta
$$




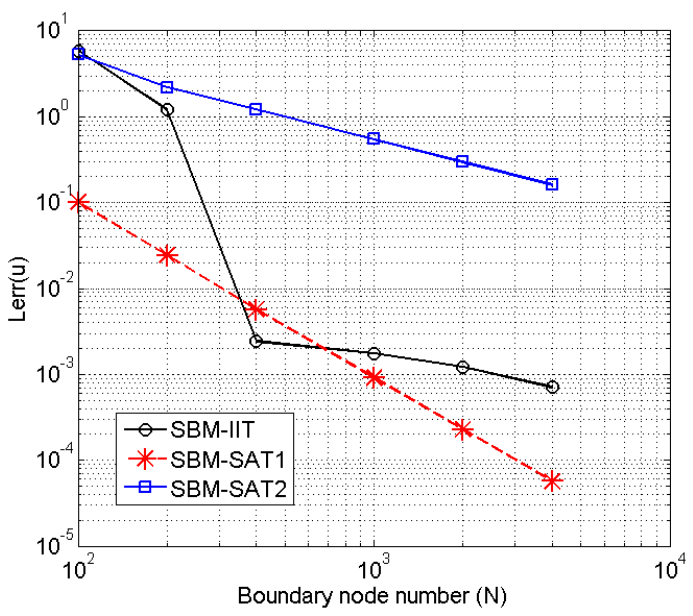

Figure 4: Convergence analysis $\operatorname{Lerr}(u)$ of the SBM with IIT, SAT1 and SAT2 for the radiation problem of a soft infinite irregular-shaped rod with $k a=1$.

Fig. 4 shows the error analysis of the SBM with three treatments for a 2D radiation problem of a soft infinite irregular-shaped rod with $k a=1$. The test points $(N T=101)$ are uniform angular distribution on the circle with radius 1.5/0.425. It has the similar conclusions to Example 1, namely, the SBM with SAT1 has the best performance among these three treatments, the SBM with SAT2 converges very slowly. Numerical stability is very sensitive to the placement of sample nodes in the SBM with IIT.

\section{Conclusions}

This study makes the numerical comparison on three treatments for calculating the source intensity factors in the singular boundary method. Numerical results show that the SBM with SAT1 provides the best performance among these three methods, and the SBM with SAT2 converges very slowly. By employing the SBM with IIT, numerical stability is very sensitive to the placement of sample nodes.

\section{References}

[1] Chen W. Singular boundary method: A novel, simple, meshfree, boundary collocation numerical method. (in Chinese). Acta Mechanica Solida Sinica 2009; 30(6): 592-99.

[2] Chen W, Fu ZJ, Wei X. Potential Problems by Singular Boundary Method Satisfying Moment Condition. CMES-Computer Modeling in Engineering \& Sciences 2009; 54(1): 65-85. 
[3] Chen W, Fu ZJ. A novel numerical method for infinite domain potential problems. Chinese Science Bulletin 2010; 55(16): 1598-603.

[4] Chen W, Wang FZ. A method of fundamental solutions without fictitious boundary. Engineering Analysis with Boundary Elements 2010; 34(5): 53032.

[5] Wei X, Chen W, Fu ZJ. Solving inhomogeneous problems by singular boundary method. Journal of Marine Science and Technology-Taiwan 2013; 21(1): 8-14.

[6] Fu ZJ, Chen W. A novel boundary meshless method for radiation and scattering problems. In: Zhang CZ, Aliabadi MH, Schanz M, editors. Advances in Boundary Element Techniques XI. Berlin, Germany: EC Ltd, United Kingdom; 2010; 83-90.

[7] Gu Y, Chen W, Zhang C-Z. Singular boundary method for solving plane strain elastostatic problems. International Journal of Solids and Structures 2011; 48(18): 2549-56.

[8] Gu Y, Chen W, Zhang J. Investigation on near-boundary solutions by singular boundary method. Engineering Analysis with Boundary Elements 2012; 36(8): 1173-82.

[9] Fu ZJ, Chen W, Gu Y. Burton-Miller-type singular boundary method for acoustic radiation and scattering. Journal of Sound and Vibration 2013: submitted.

[10] Fu ZJ, Chen W, Chen CS. Singular boundary method for radiation and wave scattering: Numerical aspects and applications. Proc. 23rd International Congress of Theoretical and Applied Mechanics (ICTAM2012) 2012.

[11] Fu ZJ, Chen W, Lin J. Improved Singular Boundary Method for Various Infinite-domain Wave Applications. Proc. Global Chinese Workshop in conjunction with 10th National Conference on Computational Methods in Engineering 2012.

[12] Gu Y, Chen W, He XQ. Domain-decomposition singular boundary method for stress analysis in multi-layered elastic materials. CMC: Computers Materials \& Continua 2012; 29(2): 129-54.

[13] Kirkup S. The boundary element method in acoustics: Integrated Sound Software; 1998.

[14] Young DL, Chen KH, Lee CW. Novel meshless method for solving the potential problems with arbitrary domain. Journal of Computational Physics 2005; 209(1): 290-321.

[15] Ochmann M. The full-field equations for acoustic radiation and scattering. The Journal of the Acoustical Society of America 1999; 105(5): 2574-84.

[16] Sarler B. Solution of potential flow problems by the modified method of fundamental solutions: Formulations with the single layer and the double layer fundamental solutions. Eng Anal Bound Elem 2009; 33(12): 1374-82.

[17] Chen JT, Chen CT, Chen PY, Chen IL. A semi-analytical approach for radiation and scattering problems with circular boundaries. Computer Methods in Applied Mechanics and Engineering 2007; 196(25-28): 275164. 